\section{rev Psi}

Revista de Psicología (UNLP)

https://revistas.unlp.edu.ar/revpsi

\title{
Validación de la escala de percepción de riesgo ante el VIH (EPR-VIH) en hombres
}

\author{
Ricardo Sánchez Medina' María Fernanda Lozano Quiroz ${ }^{1}$ \\ Oscar Iván Negrete Rodríguez ${ }^{1}$ David Javier Enríquez Negrete ${ }^{1}$ \\ Ma. de los Ángeles Estrada Martínez ${ }^{1}$ \\ Correspondencia \\ ricardo.sanchez@iztacala.unam.mx \\ Filiaciones institucionales \\ ${ }^{1}$ Facultad de Estudios Superiores Iztacala. \\ Universidad Nacional Autónoma de México \\ (UNAM, México)
}

\section{Resumen}

El objetivo de la presente investigación fue desarrollar y evaluar las propiedades psicométricas de una escala orientada a medir la percepción de riesgo ante el VIH en hombres. Participaron 1008 hombres con una edad promedio de 31 años, la mitad de ellos con diagnóstico de VIH. Se diseñó y aplicó una escala en formato tipo likert sobre la percepción de riesgo ante la transmisión del VIH por vía sexual (EPR-VIH). El análisis factorial exploratorio arrojó tres factores, prácticas sexuales de riesgo, situaciones de transmisión de VIH y prácticas sexuales seguras que en su conjunto tienen una varianza explicada de 44.90 y una consistencia interna de .78; además se encontró una validez concurrente entre la escala y la consistencia en el uso del condón. Se discuten los resultados en términos de la importancia de contar con instrumentos válidos y confiables sobre percepción de riesgo ante el VIH en hombres.

\section{Palabras clave}

percepción de riesgo | VIH | hombres | validez | confiabilidad

\section{Cómo citar}

Sánchez Medina, R., Lozano Quiroz, M. F,

Negrete Rodríguez, O. I.., Enríquez Negrete, D. J. y Estrada Martínez, M. A. (2021). Validación de la escala de percepción de riesgo ante el VIH (EPRVIH) en hombres. Revista de Psicología, 20(2), 34-54. HTTPS://DX.DOI.ORG/

\section{Proceso editorial}

Recibido

25 feb. 2021

Aceptado

27 jun. 2021
1 ra decisión

14 jun. 2021

Publicado

27 jun. 2021
ISSN

2422-572X

Licencia

Licencia de Cultura Libre CC-BY 4.0

(Compartir - Adaptar - Atribuir)

Entidad editora

RevPsi es una publicación de la Facultad de Psicología (Universidad

Nacional de La Plata, Argentina) 


\section{Validação da escala de percepção de risco de HIV (EPR-HIV) em homens}

\section{Resumo}

O objetivo da presente pesquisa foi desenvolver e avaliar as propriedades psicométricas de uma escala orientada a medir a percepção de risco ante o HIV em homens. Participaram 1008 homens com uma idade média de 31 anos, metade deles com diagnóstico de HIV. Foi concebida e aplicada uma escala em formato Likert sobre a percepção do risco de transmissão do VIH por via sexual (EPR-VIH). A análise fatorial exploratória revelou três fatores, práticas sexuais de risco, situações de transmissão de HIV e práticas sexuais seguras que, no seu conjunto, têm uma variância explicada de 44.90 e uma consistência interna de .78; além disso, foi encontrada uma validade simultânea entre a escala e a consistência no uso do preservativo. Os resultados são discutidos em termos da importância de dispor de instrumentos válidos e fiáveis sobre percepção de risco perante o HIV em homens.

\section{Palavras-chave}

percepção de risco | HIV | homens | validade | confiabilidade

\section{Validation of the HIV risk perception scale (RPS-HIV) in men}

\section{Abstract}

The objective of this research was to develop and evaluate the psychometric properties of a scale aimed at measuring the perception of HIV risk in men. 1008 men with an average age of 31 years participated, half of them diagnosed with HIV. A scale in Likert format was designed and applied on the perception of risk for HIV transmission (EPRHIV). The exploratory factor analysis yielded three factors, sexual risk practices, HIV transmission situations and safe sexual practices that together have an explained variance of 44.90 and an internal consistency of . 78; in addition, a concurrent validity was found between the scale and the consistency in the use of the condom. The results are discussed in terms of the importance of having valid and reliable instruments on risk perception of HIV in men.

\section{Keywords}

risk perception $|\mathrm{HIV}|$ men $\mid$ validity | reliability 


\section{Aspectos destacados del trabajo}

- El AP y el AFE establecieron tres factores que explican el $44.90 \%$ de la varianza.

- Hubo validez concurrente entre la escala y la consistencia en el uso del condón.

- La consistencia interna de toda la escala fue de.78.

- Instrumento valido para la evaluación de percepción de riesgo ante el VIH en hombres.

Los seres humanos en su vida diaria se exponen continuamente a factores que están inmersos en el ambiente y que puede afectarlos positiva o negativamente, considerándose una habilidad el poder identificar y evitar aquellas condiciones que implican un posible daño. Esta evaluación que se realiza acerca de los riesgos va a diferir de persona a persona, ya que es un proceso subjetivo (Salvador-Ginez y Ortega-Andeane, 2018; Slovic, 1987).

Esta temática de investigación se denomina percepción de riesgo y se encarga de conocer cómo perciben los riesgos las personas -por ejemplo, los posibles desastres naturales en su localidad, como son los temblores y las erupciones volcánicaso aquellas conductas que ponen en riesgo su salud; por ejemplo, algunas prácticas alimentarias, el ejercicio de deportes extremos, el consumo de drogas, falta de higiene personal, prácticas sexuales de riesgo, entre otros (García, 2012).

Antes que nada, es importante precisar que las personas consideran como un riesgo a todo aquello que puede representar una pérdida; también aquellas amenazas a la vida o a la salud que probabilizan obtener resultados adversos (Fox, 1999; Yates y Stone, 1992). Al respecto, en la literatura se habla de dos tipos de riesgo; el primero se basa en indicadores objetivos y el segundo en las percepciones o creencias. El riesgo objetivo se basa en la valoración técnica de expertos en el tema, mientras que el riesgo subjetivo es la evaluación realizada por cualquier persona. Cabe mencionar que, desde un punto de vista psicosocial, resulta más relevante el subjetivo debido a que así se pueden conocer aquellos sesgos en la evaluación de la población; además ambos tipos de riesgo se pueden valorar desde una vertiente individual o colectiva (Slovic, 1987).

Se ha relacionado la percepción de riesgo con la subjetividad, la cultura y la sociedad, integrando aspectos políticos y económicos; la relevancia de su estudio se debe a que, con base en ella, los individuos pueden modificar o determinar sus comportamientos, de tal forma que pueden exponerse a situaciones -que probablemente tengan consecuencias negativas en su vida- y no percatarse de tal condición (Salvador-Ginez y Ortega-Andeane, 2018).

La percepción de riesgo se puede considerar un elemento central en la predicción del 
comportamiento pues, como señala Stanojlovic (2015), es una de las variables centrales en los modelos teóricos del comportamiento y de la toma de decisiones. Considerando esto, resulta importante desarrollar instrumentos de medición que brinden la posibilidad de evaluarla en diferentes poblaciones y con relación en distintos elementos, situaciones, comportamientos o estímulos, particularmente sobre la medición de la percepción de riesgo, se ha planteado que un conjunto de factores emocionales son los que condicionan esta percepción y delinean a la vez la toma de decisiones racionalizando o prediciendo un futuro incierto.

Además de lo anterior, es importante entender cómo se define el constructo; en el ámbito de la salud, la percepción de riesgo puede definirse como la vulnerabilidad que percibe un individuo al considerar que está o no en riesgo de enfermar (Lameiras et al., 2002).

De tal forma que al centrarse en problemas de salud -específicamente en problemas de salud sexual provocados por infecciones de transmisión sexual (ITS), como lo es el VIH-, es primordial conocer las actitudes, conocimientos, creencias, juicios y las disposiciones culturales y sociales, así como la manera de actuar ante los riesgos. Es por ello que la OMS (2019) señala que, de no tomarse en cuenta estos aspectos, las iniciativas estarán destinadas al fracaso, pues debe considerarse el contexto en el que se encuentran las personas, la población a la que se dirigen las acciones, así como las instituciones y especialistas que se encargaran de ello.

Hoy en día, muchas personas se exponen continuamente a situaciones sexuales riesgosas que puede tener consecuencias de salud, como lo es contraer VIH. Cabe señalar que el VIH/SIDA es un problema actual de salud pública en América Latina y el mundo, considerado así debido a que se estima que ha causado la muerte de más de 34.7 millones de personas; por tanto, es un virus que tiene un gran impacto en la salud y en la vida de la persona, además del gran gasto público que representa (ONUSIDA, 2021).

En América Latina se estima que existe un total de 2.1 millones de personas viendo con VIH, y tan sólo en 2020 se registraron un total de 110000 nuevas infecciones (ONUSIDA 2021). En el caso particular de Argentina de acuerdo con el cierre de 2020, hay un total de 136 mil personas que viven con VIH (Organización Paramericana de la Salud [OPS], 2020) y en México viven un total de 189 mil (Centro Nacional para la Prevención y el Control del VIH y el SIDA [CENSIDA], 2020); en donde más del $94 \%$ de las infecciones han sido por vía sexual y se considera que hay una epidemia concentrada debido a que más del $80 \%$ de los casos de VIH/SIDA corresponden a hombres y que, en su mayoría, se encuentran en el rango de edad de los 25 a 39 años.

De hecho, se han identificado grupos de población clave ante el VIH, entre los cuales se encuentran hombres que tienen relaciones sexuales con hombres (HSH). Esta categoría, más que centrarse en la orientación sexual, hace hincapié en la práctica sexual que se tiene ya que el riesgo que tienen de contraer VIH es 22 veces mayor en comparación con el resto de la población (CENSIDA, 2019). Esto se debe a que la 
penetración anal es una práctica muy común en este grupo y que, aunque no es exclusiva de ellos, conlleva un mayor riesgo si no se ocupa condón debido a que la mucosa del ano es fina y puede desgarrarse fácilmente, por lo que puede facilitar microsangrado en el pene y ano, posibilitando el contacto sangre-mucosa por medio del cual se transmite el virus (OMS, 2019).

Como se mencionó anteriormente, la percepción de riesgo es un predictor de las conductas de riesgo debido a que entre más vulnerables se perciban, más se adoptarán conductas sexuales seguras (Stanojlovic, 2015; Vinaccia et al., 2007). En su evaluación debe tomarse en cuenta, por un lado, que el ser humano es un ser dinámico que tiene la capacidad de crear o reducir riesgos; por otro lado, que el contexto en el que se encuentran las personas también influye y debe ser tomado en cuenta (OMS, 2019). Con base en lo anterior se puede observar las diferencias de la percepción de riesgo en distintas poblaciones.

Por ejemplo, en un estudio con universitarios se encontró que las mujeres tuvieron una mayor percepción de riesgo en comparación con los hombres, pero ellos fueron más consistentes en el uso del condón (Mendoza et al., 2013). Probablemente esto se debió a lo reportado en algunos estudios acerca de que las mujeres adoptan conductas para prevenir un embarazo, pero en menor medida para protegerse de las ITS y VIH. Esto se debe a que hay una mayor percepción de riesgo ante un embarazo, de manera que hay una mayor preferencia por lo métodos hormonales, encontrándose así que los hombres tienen un mayor uso del condón debido a que se protegieron para evitar enfermedades, mientras que las mujeres ocupaban métodos anticonceptivos $y$, en consecuencia, muchas de ellas presentaron alguna ITS (Rosales, 2019; Uribe et al., 2016).

Por sorprendente que parezca, aún sigue habiendo grandes prejuicios y desinformación acerca de la salud sexual como son desconocer las ITS y el VIH, no conocer a nadie que tuviera alguna de ellas o pensar que las ITS son enfermedades exclusivas de homosexuales y países subdesarrollados; estos argumentos incorrectos fueron mencionados como motivos por los cuales no ocupan consistentemente el condón (Olansky et al., 2020).

También se ha visto que pueden tener sexo sin protección si conocen y confían en su pareja sexual, lo cual es similar con lo reportado acerca de que tanto hombres como mujeres consideraron que el sexo sin protección con la pareja estable era seguro y con pareja ocasional peligroso ya que la estabilidad y el principio de fidelidad hace que perciban menor riesgo (Mendoza et al., 2013) o que incluso el condón no lo empleen en todo el acto sexual (Cortés et al., 2015).

De manera que estos hallazgos sugieren que las parejas heterosexuales consideran que el condón es un método para prevenir un embarazo y no para prevenir una ITS y VIH, dando como resultado que sean inconsistentes en el uso del condón, aunque también se ve afectado su uso cuando hay de por medio sentimientos como la confianza plena o el enamoramiento (Saeteros et al., 2015) o fidelidad y estabilidad con la pareja (Cortés, et al., 2015). 
También se ha encontrado que, sean hombres o mujeres y a pesar de reconocer que el condón brinda protección ante el VIH y ser sexualmente activos, ambos deciden no usarlo (Pavía-Ruz et al., 2012). Es importante recordar que la percepción de riesgos estará influenciada por la sociedad, el momento histórico y político, así como la historia personal; muestra de ello es que el VIH, desde un inicio, se ha relacionado con los hombres homosexuales. En relación con esto, en un estudio realizado recientemente se señala que las personas homosexuales y bisexuales se percibieron en un mayor riesgo de contraer VIH en comparación con los heterosexuales (Lowry et al., 2017); esto se relaciona con lo documentado acerca de que algunos HSH practican el sexo anal sin condón (PASC), lo cual expone a los individuos (Fernández-Dávila y Morales-Carmona, 2014).

Relacionado con esto último, en un estudio realizado en España se encontró que los HSH - debido a los tratamientos antirretrovirales (TAR)-, aceptaron tener encuentros sexuales riesgosos, pasando de un $9.2 \%$ a un 19.4\% (Folch et al., 2010), lo cual concuerda con lo mencionado por Folch et al. (2006) acerca de que este grupo se siente menos preocupado por la transmisión de VIH debido a la eficacia de los tratamientos de TAR, algo que también es señalado por Collins (2012) y Lozano y Domingo (2011).

Otro dato relevante encontrado es que los HSH tienen un buen nivel de información acerca del VIH-SIDA, por lo que sus conductas riesgosas no se basan en desinformación, sino en la carencia afectiva y en la discriminación en la que viven, de manera que sobrevaloran los encuentros sexuales más allá de que sea por satisfacción física (Galindo, 2018; Posada et al., 2020).

Con base en lo expuesto sobre la percepción de riesgo y su importancia en la predicción del comportamiento - particularmente de las conductas que inciden sobre la probabilidad de contagio de alguna ITS, como es el VIH-, se observa que, en función de la población evaluada, los indicadores de la percepción de riesgo es diversa; esto se torna relevante si se desea contar con instrumentos que sean culturalmente válidos, respondiendo al contexto de las personas que se desean evaluar (Martínez et al., 2006).

En el caso particular de los hombres, se observa con base en las estadísticas a nivel nacional e internacional que son ellos quienes más se infectan por VIH, por lo cual resulta relevante diseñar estrategias de intervención orientadas a la reducción de nuevas infecciones o re-infecciones por VIH, siendo la evaluación de la percepción de riesgo un factor clave en ello. De tal forma que resulta importante contar con instrumentos válidos y confiables que permitan hacer dicha evaluación. Con base en lo anterior, el objetivo de la presente investigación es desarrollar y evaluar las propiedades psicométricas de una escala orientada a medir la percepción de riesgo ante el VIH en hombres, definida como la valoración por medio de la cual un individuo identifica y reacciona a situaciones que inciden sobre la probabilidad que se transmita o se vuelva a infectar de VIH por vía sexual. 


\section{Método}

\section{Participantes}

Participaron voluntariamente 1008 hombres de la Ciudad de México que acudieron a la Clínica Especializada Condesa (CEC) que pertenece a la Secretaría de Salud de la Ciudad de México y se dedica a la atención de personas con VIH/SIDA. El muestreo mediante el cual seleccionó a quienes participaron en este estudio fue no probabilístico por conveniencia; en otras palabras, participaron aquellos hombres que se encontraban en la clínica y que aceptaron la invitación a participar en esta investigación (Otzen y Manterola, 2017). Los criterios de inclusión fueron los de ser hombres mayores de edad y haber iniciado su vida sexual. La edad de los participantes osciló entre los 18 y 74 años con una media de $31(\sigma=.827)$; la mayoría reportó ser soltero, con nivel de estudios de licenciatura o preparatoria, sin hijos y con orientación sexual homosexual; los ingresos mensuales fueron variados y, en cuanto al estado serológico, prácticamente la mitad tenía el diagnóstico de VIH. Para mayores detalles, puede consultarse la descripción general de la muestra en la Tabla 1.

\section{Instrumentos}

Se aplicó a los participantes una cédula de datos sociodemográficos e indicadores de la conducta sexual -reactivos para evaluar la consistencia en el uso de condón- y la escala de percepción de riesgo ante el VIH. A continuación, se describe cada instrumento.

\begin{tabular}{lrlr} 
& \multicolumn{2}{c}{$\%$} & $\%$ \\
\hline Estado civil & & Ingresos mensuales \\
-Soltero & 76.4 & -Menos de \$2000 & \\
-Casado & 5.9 & -De \$2001 a \$3999 \\
-Unión libre & 15.6 & -De \$4000 a \$5999 & 12.6 \\
-Otro & 2.1 & -De \$6000 a \$9999 & 16.2 \\
Nivel de estudios & & -Más de \$10000 & 20.5 \\
-Ninguno & .8 & -No sé & 29.1 \\
-Primaria & 1.6 & Estado serológico de HIV & 11.7 \\
-Secundaria & 9.9 & -Positivo & 50.5 \\
-Preparatoria & 35.6 & -Negativo & 49.5 \\
-Universidad & 44.4 & Orientación sexual \\
-Posgrado & 7.6 & -Homosexual & 71.2 \\
Hijos & & -Bisexual & 12.8 \\
-Sí & 7.5 & -Heterosexual & 14.4 \\
-No & 91.7 & & \\
\hline
\end{tabular}


Cédula de datos sociodemográficos e indicadores de la conducta sexual. Permitió obtener datos sociodemográficos (edad, estado civil, nivel de estudios, si tenían hijos, ingresos mensuales, estado serológico de VIH y orientación sexual).

Consistencia en el uso del condón. Dos reactivos para evaluar el número de veces que se han tenido relaciones sexuales en un periodo de tres meses y el número de veces que usaron condón en ese periodo, propuesto por DiClemente y Wingood (1995). Se obtuvo un índice de consistencia del uso del condón (ICUC) con valores entre cero y uno, que fue resultado de dividir la frecuencia del uso del condón entre la frecuencia de las relaciones sexuales.

Escala de percepción de riesgo ante la transmisión del VIH por vía sexual (EPR-VIH). Con base en un análisis de la literatura sobre percepción de riesgo, se diseñó una escala de 22 reactivos en escala tipo Likert con cinco opciones de respuesta que evalúan qué tan de acuerdo o no están con la afirmación. Para la construcción de la escala nos apegamos a las recomendaciones de Miguel-Tobal (2003), específicamente en lo que refiere a la estrategia denominada racional, dado que los reactivos de la escala se desarrollaron en función del modelo teórico del cual partimos (percepción de riesgo). Esto es coincidente con lo planteado por Pérez y Tornimbeni (2014), quienes plantean, como primer paso en la construcción de instrumentos de medición, definir el dominio a evaluar para posteriormente redactar los reactivos en apego a tal definición.

El modelo teórico para la construcción del instrumento fue el de Slovic (1987) referente a la percepción de riesgo, que considera la naturaleza compleja y socialmente determinada del riesgo. A lo largo de la historia se han propuesto varios paradigmas para su medición, tales como la axiomática, el socio-cultural y el psicométrico. Este último es el que nos interesa ya que toma en cuenta factores emocionales y guían la toma de decisiones humanas. En este caso, al interesarnos la percepción de riesgo ante el VIH adquirido por vía sexual, se evaluaron los riesgos personales, dado que se evaluó por medio de situaciones en las que se les involucraron a ellos y a sus parejas, de manera que se construyó la escala considerando comportamientos seguros e inseguros; los inseguros fueron divididos en prácticas sexuales inseguras en general y relacionadas con el VIH.

Cabe señalar que Slovic (1987) considera que el paradigma psicométrico ha demostrado que el riesgo percibido es cuantificable y predecible, por lo que la técnica es adecuada para identificar similitudes y diferencias entre grupos con relación a las percepciones y actitudes ante el riesgo; esto último es un de las tantas posibles aplicaciones de la escala que se propone.

\section{Tipo de estudio}

Se realizó un estudio de tipo instrumental, el cual, de acuerdo a las categorías de Montero y León (2007), se centra en el análisis de las propiedades psicométricas de una escala de medición. 


\section{Procedimiento}

La aplicación de las escalas se realizó de manera individual en las salas de espera de la Clínica Especializada Condesa de la Ciudad de México. Se invitó a participar a los hombres que se encontraban presentes y se les explicó el objetivo de la investigación señalando que esta se centraba en algunos aspectos relacionados con su vida sexual. A su vez, se hacía énfasis en la confidencialidad de su información y en el uso con fines científicos; en caso de aceptar, se les hacía entrega del cuestionario y de un lápiz. Siempre hubo al menos un investigador próximo por si surgía alguna duda y para recibir el cuestionario una vez terminaban de contestarlo. En ese momento, se les daba las gracias por su participación.

\section{Análisis de datos}

Los datos recopilados fueron analizados en el programa estadístico SPSS v.20, la base de datos se encuentra disponible en un repositorio de acceso abierto (Sánchez et al., 2021). Para medir la percepción de riesgo ante el VIH, la escala se construyó tomando en cuenta tres factores: 1. Prácticas sexuales de riesgo; 2. Situaciones de transmisión de VIH y 3. Prácticas sexuales seguras. Considerando las recomendaciones y puntualizaciones de Lloret-Segura et al. (2014), se realizó un análisis paralelo para determinar el número de factores de la escala y un análisis factorial exploraratorio (AFE) para identificar la estructura de una prueba o identificar las variables latentes presentes y preferentemente un Análisis Factorial Paralelo (AFP). Este último se recomienda dado que brinda la posibilidad de identificar y seleccionar los componentes o factores comunes que presentan valores que sobrepasan aquellos que serían atribuidos al mero azar (Horn, 1965). Dado que el análisis paralelo no es posible hacerlo en SPSS, se utilizó el programa R para ello, posteriormente se continuó el resto del análisis con SPSS, dado que el objetivo es presentar la evidencia de validez de la escala considerando las recomendaciones de Lloret-Segura et al. (2014), independientemente del programa que se use para ello.

El AFP se realizó por medio del software R versión 4.0.2. Primero se calculó una matriz de correlaciones con la función cor(); después se calcularon los valores eigen y el análisis paralelo con la función parallel(); posteriormente, se calculó el número de factores con la función $n$ Scree() y, finalmente, se obtuvo la gráfica de sedimentación.

Cuando se hace uso del Análisis Factorial, es necesario verificar la presencia de valores extremos (outliers) y si se cuenta con una distribución normal de las puntuaciones observadas en las variables consideradas para el análisis (Pérez y Tornimbeni, 2014). Para identificar si efectivamente existía normalidad en la muestra, se calcularon los índices de asimetría y curtosis -así como las pruebas de normalidad de Kolmogorov-Smirnov con corrección de Lilliefors de cada uno de los reactivos- y se realizó un histograma. También se consideró la prueba de adecuación muestral KMO y la prueba de esfericidad de Barlett, que indican si fue pertinente o no llevar a cabo un Análisis Factorial a partir de la interrelación entre los valores de 
los reactivos que integran la escala. Finalmente, para poder dar cuenta de la validez de concurrente, se correlacionó el ICUC con el puntaje total de la escala EPR-VIH y con el puntaje obtenido en cada factor identificado.

Por último, considerando lo que planean Clark-Carter (2002), Kerlinger y Lee (2002), Muñiz (1998), Olaz (2014), y Reidl-Martínez (2013) sobre lo importante que resulta contar con instrumentos que midan el atributo de interés con el menor error posible -para obtener la mayor precisión posible y siendo la confiabilidad la propiedad de los instrumentos de medición que da cuenta del error de medida-, se decidió analizar la consistencia interna de cada uno de los factores del instrumento a partir del cálculo y análisis del coeficiente alfa de Cronbach.

\section{Resultados}

Tras analizar la forma de la distribución de los datos recopilados se identificó que la media de cada uno de los ítems de la escala EPR-VIH osciló entre 3.31 y 4.52, siendo el puntaje mínimo 1 y el máximo 5 (ver tabla 2). Once de los ítems obtuvieron una media alta; esto significa que los participantes presentaron una percepción de riesgo alta ante la transmisión del VIH por vía sexual y las desviaciones estándar de los ítems oscilaron entre .85 y 1.52 .

Con relación a la forma de la distribución de los valores, en la Tabla 2 se presentan los índices de asimetría y curtosis. Los índices de 4 de los 16 ítems se encuentran fuera de los rangos de valores +/- 2, lo que significa que algunos de los ítems no presentaron una distribución normal. Los valores obtenidos en las pruebas de normalidad de Kolmogorov-Smirnov con corrección de Lilliefors no cumplen con el valor que establece el criterio de normalidad -a pesar de encontrarse correlaciones positivas la significancia estadística ha sido $.001-\mathrm{y}$, por tanto, la escala no tiene una distribución normal. En cuanto a la correlación ítem-total, se puede observar que todas las correlaciones fueron positivas y significativas, por lo que no se descartó ningún elemento en esta primera etapa.

Al realizar el análisis de las frecuencias de los puntajes totales de la EPR-VIH, se encontró una distribución que se asemeja a la normal, esto se puede observar en la Figura 1.

Los valores obtenidos en la prueba de adecuación muestral dan cuenta de un KMO de .82. Esto significa que las correlaciones parciales entre las variables permiten afirmar que fue pertinente usar el análisis factorial para analizar la EPR-VIH; asimismo, los valores obtenidos en la prueba de esfericidad de Barlett fueron significativa $\left(x^{2}=3186.90, g l=120, p<.001\right)$. Ambos resultados permiten concluir que los ítems tienen una intercorrelación por lo que es pertinente realizar un análisis factorial. Posteriormente se realizó el AFP para confirmar el número de factores a extraer; en la Figura 2 se observa que, de acuerdo con el cruce entre los valores, el número adecuado para hacer el análisis es tomar en cuenta tres factores. 


\begin{tabular}{|c|c|c|c|c|c|c|}
\hline Ítem & Media & $\mathrm{DE}$ & Asimetría $^{1}$ & Curtosis $^{2}$ & $\begin{array}{l}\text { Kolmogorov-Smirnov } \\
\text { con corrección de Lilliefors }\end{array}$ & $\begin{array}{l}\text { Correlación } \\
\text { ítem-total }\end{array}$ \\
\hline 1. & 4.00 & 1.35 & -1.11 & -.10 & $.32^{\star *}$ & ${ }^{\star} \quad .46^{\star \star}$ \\
\hline 2. & 4.07 & 1.27 & -1.25 & .38 & $.31^{* *}$ & $.49^{* *}$ \\
\hline 3. & 4.42 & 1.01 & -2.03 & 4.06 & $.35^{* *}$ & $.42^{\star *}$ \\
\hline 4. & 4.45 & 1.08 & -2.02 & 3.47 & $.39^{* *}$ & $.44^{\star \star}$ \\
\hline 5. & 3.34 & 1.52 & -.31 & -1.36 & $.21^{* *}$ & $.48^{\star *}$ \\
\hline 6. & 4.19 & 1.23 & -1.48 & 1.02 & $.34^{* *}$ & $.56^{\star *}$ \\
\hline 7. & 4.26 & 1.21 & -1.62 & 1.49 & $.36^{* *}$ & $.28^{\star *}$ \\
\hline 8. & 4.45 & 1.17 & -2.08 & 3.36 & $.42^{* *}$ & $.31^{\star *}$ \\
\hline 9. & 3.18 & 1.40 & -.20 & -1.14 & $.15^{* *}$ & $.37^{* *}$ \\
\hline 10. & 4.52 & 1.03 & -2.42 & 4.90 & $.41^{\star *}$ & $.49^{* *}$ \\
\hline 11. & 4.01 & 1.28 & -1.12 & .08 & $.30^{* *}$ & $.49^{* *}$ \\
\hline 12. & 3.58 & 1.37 & -.60 & -.79 & $.20^{* *}$ & $.38^{\star *}$ \\
\hline 13. & 3.31 & 1.42 & -.23 & -1.18 & $.19^{* *}$ & $.49^{* *}$ \\
\hline 14. & 3.93 & 1.28 & -1.03 & -.06 & $.27^{* *}$ & $.45^{\star *}$ \\
\hline 15. & 4.08 & 1.19 & -1.20 & .48 & $.29^{* *}$ & $.51^{\star \star}$ \\
\hline 16. & 4.62 & .85 & -.29 & 1.27 & $.36^{* *}$ & $.36^{\star *}$ \\
\hline
\end{tabular}

Tabla 2. Análisis de los ítems de la escala EPR-VIH

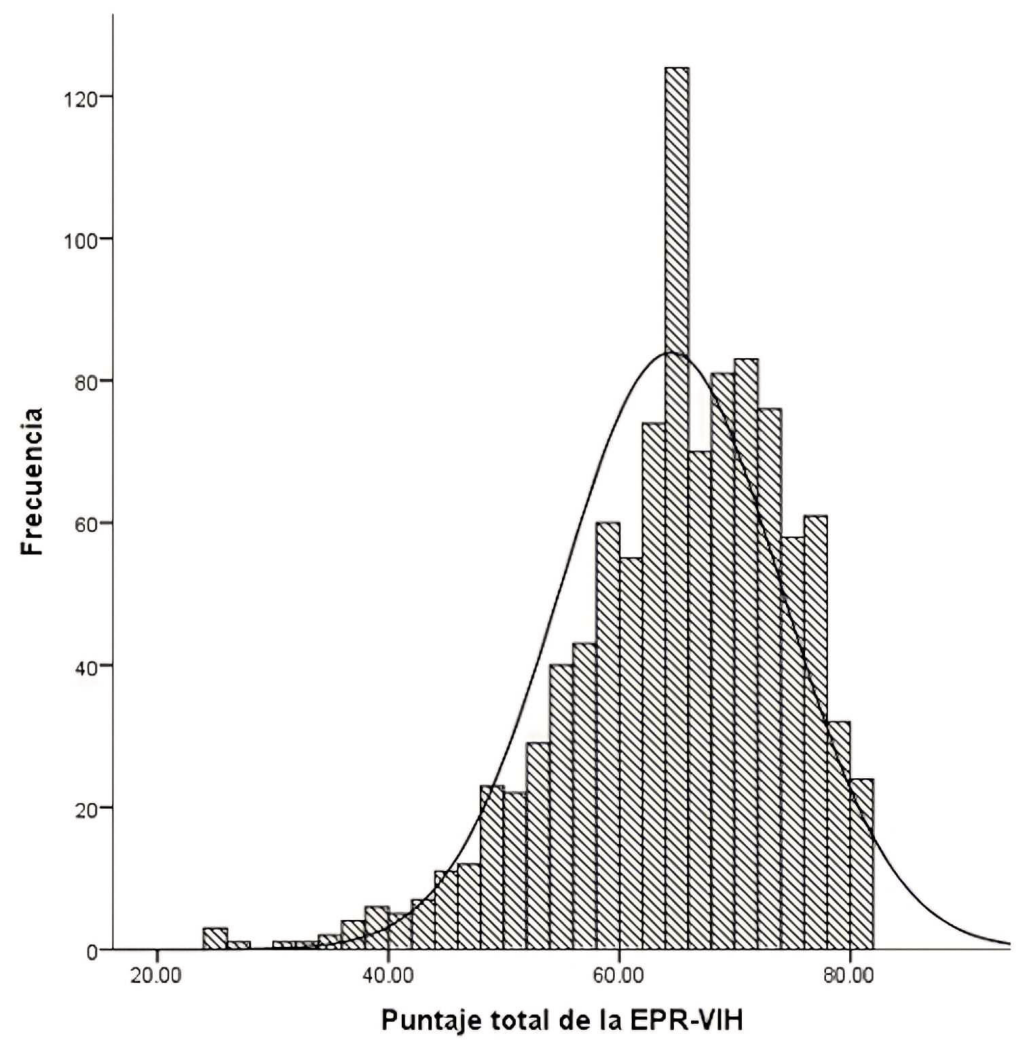

Figura 1. Distribución de la escala EPR-VIH 


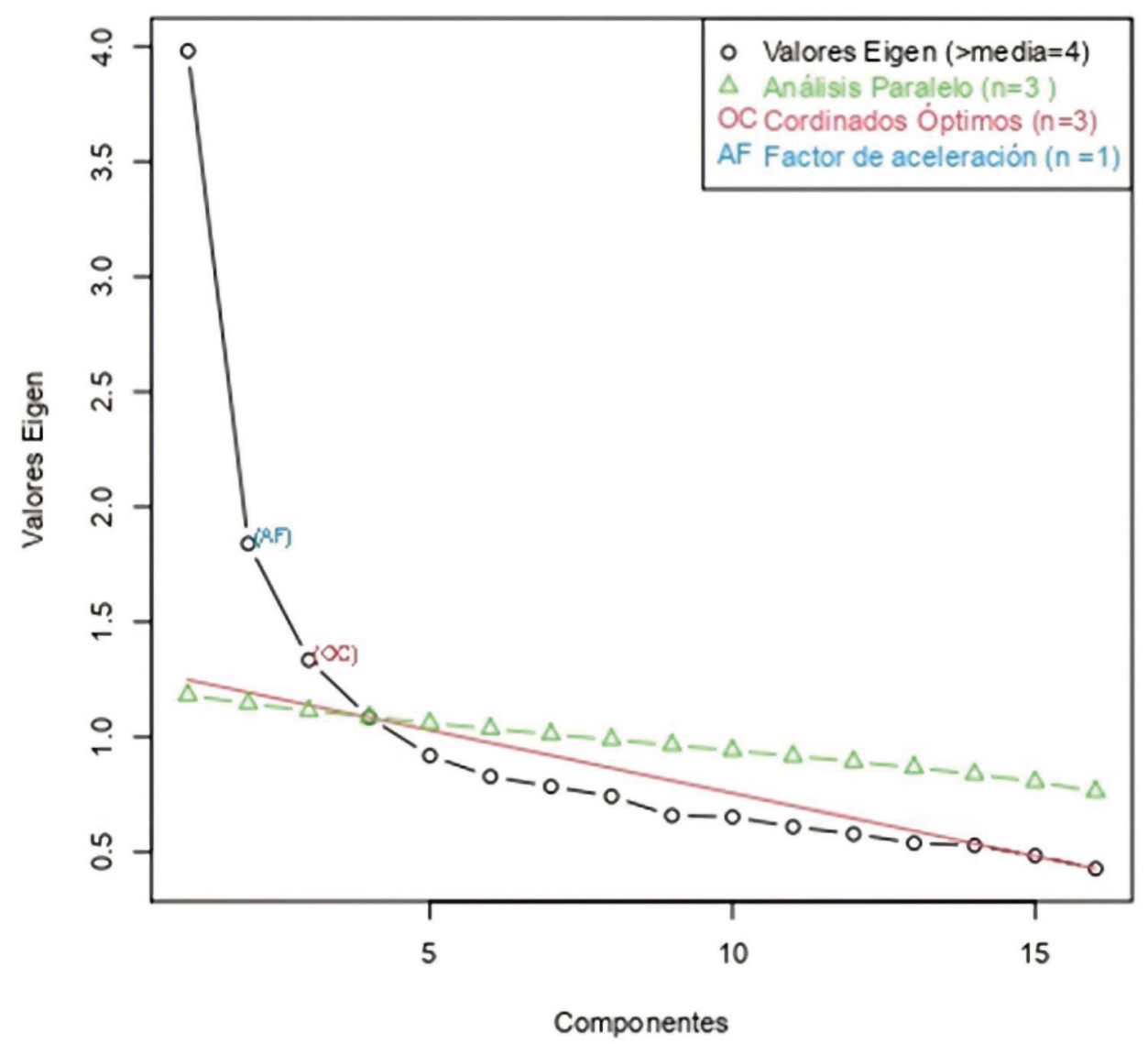

Figura 2. Análisis paralelo para los factores de la escala EPR-VIH

En la versión final de la escala, se presentan en la Tabla 3 tres factores que explican el 44.9 de la varianza total. Estos factores son: prácticas sexuales de riesgo, situaciones de transmisión de VIH y prácticas sexuales seguras. Las cargas factoriales observadas de todos los ítems son mayores a .45 .

En la Tabla 3 también se puede observar que se calculó el alfa de Cronbach para cada uno de los factores identificados: el factor 1 obtuvo un alpha de .70, el factor dos .72 y el factor tres .58. En conjunto, la escala obtuvo un alfa de.78.

A su vez, el análisis factorial es evidencia de que cuenta con validez de constructo el instrumento. Para probar la validez concurrente en la escala de EPR-VIH se correlacionó el puntaje total de la escala con el ICUC, ya que la evaluación de las situaciones riesgosas es un predictor del comportamiento sexual y de la toma de decisiones, de tal manera que la percepción de riesgo ante la infección y/o reinfección del VIH está relacionada con el uso consistente del condón (ver Tabla 4). 
Número de reactivo y factores

\section{Carga factorial}

1

2

\section{Factor 1. Prácticas sexuales de riesgo}

8. Tener sexo anal sin protección se puede considerar una práctica de sexo seguro ante la transmisión del VIH

7. El coito interrumpido se puede considerar una práctica de sexo seguro ante la transmisión del VIH

4. En un encuentro sexual con una pareja informal se pueden tener relaciones sin usar condón dado que no hay ningún peligro

16. Aceptar un encuentro sexual sin protección tras consumir alguna droga es una práctica de sexo seguro

10. En caso de que una persona se haya infectado de VIH puede tener sexo sin protección sin preocuparse por este virus

\section{Factor 2. Situaciones de transmisión de VIH}

13. Si mi pareja y yo tomamos antirretrovirales podemos tener relaciones sexuales sin protección

5. Es seguro tener relaciones sin protección con una persona que tiene VIH si debido al tratamiento se encuentra indetectable el virus

11. Si el resultado de la prueba de VIH de uno de los miembros de la pareja resulta negativo se pueden tener relaciones sexuales sin protección

14. Si el resultado en la prueba de VIH fuera negativo es seguro tener relaciones sexuales sin condón

2. Es posible practicar sexo sin protección sin preocuparse por las consecuencias

15. Me siento tranquilo al iniciar una relación sexual sin condón

\section{Factor 3. Prácticas sexuales seguras}

3. Usar de manera consistente y correcta el condón previene que contraiga VIH

9. Pospongo un encuentro sexual con mi pareja formal cuando no cuento con condones para prevenir cualquier peligro

12. Llevo siempre conmigo condones por cualquier situación que pueda presentarse

6. Es indispensable usar condón con un portador de VIH

1. Los contactos sexuales sin condón deben de evitarse si algún miembro de la pareja es portador de VIH

\begin{tabular}{lrrr}
\hline Varianza Explicada por factor: & 24.9 & 11.5 & 8.3 \\
Alfa de Cronbach por factor: & .70 & .72 & .58 \\
Varianza explicada total: & 44.90 & & \\
Alfa de Cronbach total: & .78 & & \\
\hline
\end{tabular}




\begin{tabular}{lllll}
\hline & $\mathbf{1}$ & $\mathbf{2}$ & $\mathbf{3}$ & 4 \\
\hline 1. Índice de consistencia del uso del condón (ICUC) & & & \\
2. Factor 1. Prácticas sexuales de riesgo & .00 & & \\
3. Factor 2. VIH y omisión del uso del condón & $.22^{\star *}$ & $.38^{\star *}$ & \\
4. Factor 3. Prácticas sexuales seguras & $.35^{\star \star}$ & $.16^{\star *}$ & $.38^{\star \star}$ & \\
\hline Nota. ${ }^{* *}$ La correlación es significativa al nivel .01 (bilateral). & & \\
\hline
\end{tabular}

Tabla 4. Correlaciones entre el ICUC y la escala EPR-VIH

Los resultados mostraron correlaciones positivas y significativas en dos factores. Esto significa que, a mayor percepción de riesgo, mayor será la probabilidad de que la persona sea consistente en el uso del condón. Cabe señalar que este análisis se realizó únicamente con 788 casos de lo 1008, dado a que sólo estos habían tenido relaciones sexuales en los últimos tres meses, dato indispensable para calcular el ICUC.

\section{Discusión}

A partir de los hallazgos encontrados en esta investigación, se ratifica lo descrito por Fox (1999), Salvador-Ginez y Ortega-Andeane (2018), Slovic (1987), y Yates y Stone (1992) en relación a la complejidad que representan el estudio y la medición de la percepción de riesgo al combinar elementos objetivos y subjetivos, en donde se considera la probabilidad de ocurrencia de una situación adversa a partir de la evaluación personal.

Además, dado que la percepción de riesgo incluye aspectos cognitivos -como las actitudes, creencias, valores, sentimientos, juicios de las personas- e intervienen disposiciones sociales y culturales que afectan la manera en que el individuo enfrenta y determina las acciones necesarias para evitar una consecuencia adversa -así como también la historia previa constituye un aspecto relevante en este tema, tal como señala Stanojlovic (2015)-, la construcción de los ítems resulta una tarea compleja, pues todos los elementos mencionados tienen que ser representados de forma integral y sintética.

Se debe recordar que resulta importante contar con instrumentos confiables y válidos que midan la percepción de riesgo ante el contagio del VIH, dado que las personas aceptan exponerse a ciertos riesgos a partir de juicios de valor sesgados sobre una situación de peligro, por tanto, se requiere el análisis de variables y procesos para conocer por qué las personas restan importancia a riesgos que perjudican su integridad. Por lo anterior es probable algunas personas subestimen o acepten riesgos, por lo que contar con un instrumento que evalúe la percepción de riesgo ante el VIH brinda la posibilidad de identificar uno de los factores que propician una evaluación incorrecta ya que -de acuerdo con lo señalado por 
Stanojlovic (2015) y Vinaccia et al. (2007), concebirse vulnerable tiene como consecuencia un cambio en el comportamiento que probabiliza positivamente la presencia de conductas de protección, como es el caso de postergar un encuentro en caso de no contar preservativos o usar el condón en sus prácticas sexuales.

Como se mencionó en un principio, el objetivo del presente trabajo fue evaluar las propiedades psicométricas de una escala desarrollada para medir la percepción de riesgo ante el VIH, la cual está conformada por 16 ítems. Vale la pena recordar que la validación o evaluación de una escala es un proceso complejo, continuo y extenso. En primer lugar, acatando las recomendaciones de varios autores (CampoArias et al., 2012; Nunnally y Berstein, 1995) se obtuvo la confiabilidad y, posteriormente, la validez.

Previo a esto se consideraron: 1. Las pruebas de asimetría y curtosis (Bandalos y Finney, 2010); 2. Las pruebas de normalidad de Kolmogorov-Smirnov, con corrección de Lilliefors de cada uno de los reactivos e histograma (Pérez y Tornimbeni, 2014); 3. La prueba de adecuación muestral KMO y 4. La prueba de esfericidad de Barlett (Campo-Arias et al., 2012; Hogarty et al., 2005; Vallejo, 2011), en tanto son indicadores que permiten conocer si es conveniente realizar un análisis factorial; en este caso, todos las pruebas justifican la decisión de realizarlo.

Con base en las recomendaciones de Horn (1965) y Lloret-Segura et al. (2014), se empleó el análisis paralelo para estimar el número de componentes de la escala. En este caso fueron 3 factores; con este dato se realizó un análisis factorial en el SPSS para conocer la composición de cada componente ya que, aunque previamente la escala fue pensada en estos tres factores, se buscó reafirmar la estructura de los componentes del instrumento. De manera general se observa que el análisis factorial per se es una manera de demostrar que la escala cuenta con validez de constructo. Tal como comenta Campo-Arias et al. (2012) y Hogarty et al. (2005), el AF demuestra, de forma matemática, que la construcción teórica de la percepción de riesgo se refleja en el patrón de respuesta de este grupo.

También se exploró la validez predictiva con el ICUC; dicha propuesta se basa en la revisión de la literatura (Colins, 2012; Galindo, 2018; Lozano y Domingo, 2011; OMS, 2019), pero se encuentra que el factor 1 tiene una correlación nula y los otros dos factores tienen correlaciones bajas, aunque significativas. Por ello se recomienda explorar si en otras poblaciones podría ser el ICUC un indicador adecuado o si el ICUC debe tomarse en cuenta con base en el tipo de práctica sexual ya que, en este caso, el índice se calculó sin tomar en cuenta esto último.

Hablando particularmente de los tres factores de la escala, se puede afirmar que estos son sólidos, dado que se componen por más de tres ítems y cada uno de los ítems tiene un coeficiente mayor de .40 (Campo-Arias et al., 2012; Hogarty et al., 2005; Vallejo, 2011) Aunque se debe tomar en cuenta los valores de la consistencia interna, la escala en su conjunto cuenta con propiedades psicométricas adecuadas que harían viable su utilización en conjunto. 
Respecto al análisis de confiabilidad por factor, se observa que los primeros dos factores cuentan con un alfa mayor vs. el tercero, es necesario tomar en cuenta lo que señalan diversos autores respecto a qué valores se consideran aceptables (.70, $.72>.58)$. Por ejemplo, Vallejo (2011) considera que un alfa a partir de .60 es aceptable y a partir de .80 muy bueno, mientras que Nunnally y Berstein (1995), Olaz (2014) y Reidl-Martínez (2013) mencionan que .70 es suficiente cuando un test que sólo se utiliza con fines de investigación, pero siempre es recomendable un índice de .80; aunque si los test serán empleados para la toma de decisiones que afectan a las personas examinadas (con fines diagnósticos), es necesario un índice de .90. Como se puede observar, parece que, salvo el tercero, los dos primeros factores cuentan con niveles aceptables.

Este factor se refiere a las prácticas sexuales seguras, por lo que una explicación de por qué probablemente no se cuenta con un alpha mayor en el último factor puede deberse a las particularidades de la población. Tal como se mencionó anteriormente, se trabajó en una clínica especializada de atención a personas con VIH; quienes tenían un diagnóstico de VIH o eran hombres que acudian a solicitar una prueba de detección del VIH, de los cuales cerca del $88.9 \%$ reportaron haber tenido relaciones sexuales con alguien de su mismo sexo. De acuerdo con Baruch (2014) y Bastida, (2015), esta población se caracteriza por "vivir el momento", "tener sexo de verdad" sin uso de condón en sus relaciones sexuales (bareback), por lo que surge la necesidad de evaluar en otras muestras de hombres, en diferentes contextos. Inclusive tomando en cuenta el tipo de pareja sexual, algunos autores coinciden en que el uso del condón es más frecuente cuando se tienen relaciones sexuales con pareja ocasional que con una pareja regular (Huang et al., 2020; Milic et al., 2020).

Con base en lo anterior, es recomendable seguir investigando las propiedades psicométricas de la escala, evaluar en muestras similares y mostrar otras fuentes de validez y confiabilidad para tener mayor certeza de que se está evaluando el constructo (Lloret-Segura et al. 2014; Martínez et al. 2006).

A modo de conclusión, estos hallazgos coinciden en términos teóricos con lo planteado por Salvador-Ginez y Ortega-Andeane (2018), Slovic (1987) y Stanojlovic (2015) referente a que la evaluación de la situación de riesgo a partir de signos físicos $\mathrm{y}$ sociales, permite diferenciar lo amenazante de lo seguro y, por ende, probablemente afecta el comportamiento emitido.

La validación y confiabilidad de la presente escala muestra la pertinencia de la aplicación de misma en población varonil del área metropolitana de la Ciudad de México a fin de tener por objetivo predecir el comportamiento del uso del condón, particularmente de que sean consistentes. Esto es relevante dado que es la conducta recomendada para salvaguardar la salud sexual de la población sexualmente activa, independientemente del estado serológico de VIH (ONUSIDA, 2019; CENSIDA, 2019a). De tal forma que si deseara evaluar a mujeres sería necesario volver a presentar envidencias de validez, ya que como lo mencionan Mendoza et al. (2013), la percepción de riesgo es diferente entre hombres y mujeres. 
Por último, a partir de los análisis realizados a la escala EPR-VIH, se puede decir que cuenta con evidencia empírica de sus propiedades psicométricas, por lo que es un instrumento que permite conocer la percepción de riesgo ante la infección o reinfección de VIH por vía sexual en hombres. De tal forma que, con base en los resultados de la escala, podrá permitir el diseño estrategias que busquen la concientización del riesgo de las conductas sexuales de los evaluados.

\section{Limitaciones y futuras líneas de investigación}

$\mathrm{Al}$ reflexionar sobre los hallazgos y las dificultades que se presentaron a lo largo de la investigación aquí descrita, surgen una serie de ideas que a continuación se exponen. Con relación a la conformación de la muestra, sería conveniente que en futuras investigaciones se cuente con una mayor variedad de perfiles de los participantes, esto derivado a que esta investigación se llevó a cabo en una clínica que se especializa en el diagnóstico y tratamiento del VIH-SIDA y cuya población cautiva cuenta con un perfil particular clase media, predominando entre los participantes aquellos con estudios de nivel medio superior, superior y posgrado.

Estas características les dan la posibilidad de preocuparse y estar al pendiente de salud ya que, aquellos sin tener diagnóstico previo se acercaron a la institución para realizarse la prueba y quienes ya contaban con un diagnóstico positivo, se encontraban dando seguimiento a su atención médica. Por lo tanto, a partir de tal condición, una limitación importante es la posibilidad de generalizar los hallazgos de la escala desarrollada al no contar con una muestra representativa de la población de interés.

A su vez, sería recomendable para futuras investigaciones aplicar la escala en otros contextos realizando los ajustes pertinentes en relación de variaciones lingüísticas e incluso prácticas en función de variables contextuales, como puede ser la región del país e incluso aspectos culturales particulares del subgrupo al que pertenezcan los participantes. En este último punto, es pertinente considerar que no hay una homogeneidad de estilos, prácticas y usos del lenguaje entre la comunidad de hombres que tienen sexo con hombres, mucho menos en sus motivaciones para llevar a cabo esta práctica.

Conviene finalmente explorar en un futuro la relación que existe entre la percepción de riesgo y el uso del condón asociadas a las diferentes prácticas sexuales, y el tipo de pareja (ocasional o formal) ya que, en la literatura se ha observado que son variables que tienen un papel importante en la toma de decisiones. A partir de esto conviene que las intervenciones que se planeen pongan énfasis en la percepción de riesgo puesto que, a partir de la validez concurrente encontrada en este estudio, se identificó su relación con el uso del condón.

\section{Implicaciones sociales y políticas}

Dada la relevancia social y en términos de las políticas de salud por la relevancia de un tema como es la transmisión del VIH y su evolución hacia el SIDA, en caso de no ser atendido, resulta de suma importancia el desarrollar instrumentos de evaluación 
que puedan dar una radiografía lo más precisa posible de las conductas que presentan los individuos mayormente vulnerables a la transmisión o re-transmisión del virus, esto para disminuir las situaciones de riesgo a las que se exponen y en contraparte promover los comportamientos que se han calificado como protectores o seguros.

Otra de las de las implicaciones sociales para dar seguimiento e incluso la atención necesaria a la población de interés para esta investigación, y que socialmente en sí misma es blanco de diversos estudios sobre el tema, es que en función de los hallazgos obtenidos se evidenció que existe ya una cultura de la prevención y el cuidado de la salud. A su vez, es valiosa esta la información ya que, el perfil de los participantes de esta investigación ha sido identificado como de alto riesgo ante la transmisión por VIH debido a las prácticas sexuales anales y que en muchos casos se lleva a cabo sin protección, recordemos que el ano es un esfínter rígido a la penetración y puede que ocurra un microsangrado que propicia el cambio de fluidos, lo cual probabiliza que haya una transmisión en caso de que alguno de los involucrados tenga VIH.

\section{Agradecimientos}

Esta investigación fue realizada gracias al Programa UNAM-PAPIIT IN309719.

\section{Referencias}

Bandalos, D. L. y Finney, S. J. (2010). Factor analysis: Exploratory and confirmatory. En G. R. Hancock y R. O. Mueller (Eds.), Reviewer's guide to quantitative methods (pp. 93-114). Routledge.

Baruch, R. (2014). La epidemia del VIH entre hombres que tienen sexo con hombres en México: el caso del bareback. Espolea.

Bastida, L. (2019). PrEP: las siglas de la prevención. HT TPS:// MICROADMIN.JORNADA.COM.MX/ LETRAESE/2019/12/04/PREP-LAS-SIGLAS-DE-LAPREVENCION-4919.HTML

Campo-Arias, A., Herazo, E. y Oviedo, H. (2012) Análisis de factores: fundamentos para la evaluación de instrumentos de medición en salud mental. Revista Colombiana de Psiquiatría, 42(3), 659-671. HTTPS://DOI.ORG/10.1016/S0034-7450(14)60036-6
CENSIDA [Centro Nacional para la Prevención y el Control del VIH y el Sida] (2019). Día mundial del SIDA. HTTPS://WWW.GOB.Mx/CMs/UPLOADS/ A T T A C H M E T/F I L E $513718 /$ BOLETIN_D_A_MUNDIAL_28_NOV_VFIN.PDF

CENSIDA [Centro Nacional para la Prevención y el Control del VIH y el Sida] (2020). Sistema de vigilancia epidemiológica de VIH. Informe histórico al 4to. Trimesre de VIH 2020. нтTPs:// WWW.GOB.MX/CMS/UPLOADS/ATTACHMENT/FILE/ 622468/VIH-SIDA_4TOTRIM_2020.PDF

Clark-Canter, D. (2002). Las etapas preliminares de la investigación. En D. Clark-Canter (Ed.), Investigación cuantitativa en psicología. Del diseño experimental al reporte de investigación (pp. 18-33). Oxford University Press. 
Collins, S. (2012). Risk of HIV reinfection may be similar to risk of initial HIV infection. Conference on Retroviruses and Opportunistic Infections CROI, 13(3), 22-23.

Corralejo-Dios, H. y Pérez, C. (2007). Normas para el desarrollo y revisión de estudios instrumentales: consideraciones sobre la selección de test en la investigación psicológica. International Journal of Clinical and Health Psychology, 7(3), 863-882.

Cortés, A., García, R. y Ochoa, S. (2015). Comportamiento sexual y uso del condón en estudiantes de la Facultad de Tecnología de la Salud. Revista Cubana de Medicina, 67(2), 202-212.

DiClemente, R. y Wingood, G. (1995). A randomized controlled trial of an hiv sexual risk reduction intervention for young african-american women. Journal of the American Medical Association, 274(16), 1271-1276. HTTPS:// DOI.ORG/10.1001/JAMA.1995.03530160023028

Fernández-Dávila, P. y Morales-Carmona, A. (2014) "Me olvidé que tenía el VIH": motivos para tener penetración anal sin condón en hombres VIHpositivos que tienen sexo con hombres en España. Revista Multidisciplinaria del SIDA, 1(2), 7-24.

Folch, C., Casabona, J., Muñoz, R., González, V. y Zaragoza, K. (2010). Incremento en la prevalencia del VIH y en las conductas de riesgo asociadas en hombres que tienen sexo con hombres: 12 años de encuestas de vigilancia conductual en Cataluña. Gaceta Sanitaria de Barcelona, 24(1), 40-46. HTTPS://DOI.ORG/10.1016/J.GACETA.2009.06.010

Folch, C., Marks, G., Esteve, A., Zaragoza, K. y Muñoz, R. (2006). Factors associated with unprotected sexual intercourse with steady male, casual male, and female partners among men who have sex with men in Barcelona, Spain. AIDS Education and Prevention, 18(3), 227-242. HTTPS://DOI.ORG/ 10.1521/AEAP.2006.18.3.227

Fox, N. (1999). Postmodern reflections on 'risk', 'hazards' and life choice. En D. Lupton (Ed.), Risk and sociocultural theory: New directions and perspectives (pp. 12-33). HTTPS://DOI.ORG/ $10.1017 / \mathrm{CBO} 9780511520778$

Galindo, M. (2018). Diagnóstico precoz del VIH: un reto importante. Revisión y reflexiones sobre el tema. Revista Multidisciplinaria del SIDA, 6(13), 39-56.

García, J. (2012). Concepto de percepción de riesgo y su repercusión en las adicciones. Salud y Drogas, 12(2), 133-151. HTTPS://DOI.ORG/10.21134/HAAJ.V12I2.2
Hogarty, K., Hines, C., Kromrey, J., Perron, J. y Mumford, A. (2005). The quality of factor solutions in exploratory factor analysis: The influence of sample size, communality, and overdetermination. Educational and Psychological Measurement, 65(2), 202-226. HTTPS://DOI.ORG/ $10.1177 / 0013164404267287$

Horn, J. (1965). A rationale and test for the number of factors in factor analysis. Psychometrika, 30, 179-185. HTTPS://DOI.ORG/10.1007/BFO2289447

Huang, Y., Yu, B., Jia, P., Wang, Z., Yang, S., Tian, C., Lai, W. y Yang, S. (2020). Association between psychological factors and condom use with regular and nonregular male sexual partners among Chinese MSM: A quantitative study based on the health belief model. BioMed Research International, 20, artículo 5807162, 1-10. HTтPS:// DOI.ORG/10.1155/2020/5807162

Kerlinger, F. y Lee, H. (2002). Confiabilidad. En F. Kerlinger y H. Lee (Eds.), Investigación del comportamiento. Métodos de investigación en las ciencias sociales (pp. 581-602). Mc Graw Hill.

Lameiras, M., Rodríguez, Y. y Dafonte, S. (2002). Evolución de la percepción de riesgo de la transmisión heterosexual del VIH en universitarios/as españoles/las. Psicothema, 14(2). 255-261.

Lloret-Segura, S., Ferreres-Traver, A., HernándezBaeza, A. y Tomás-Marco, I. (2014). El análisis factorial exploratorio de los ítems: una guía práctica, revisada y actualizada. Anales de Psicología, 30(3), 1151-1169. HTTPs://DOI.ORG/ 10.6018/ANALESPS.33.2.270211

Lowry, R., Dunville, R., Robin, L. y Kann, L. (2017). Early sexual debut and associated risk behaviors among sexual minority youth. American Journal of Preventive Medicine, 52(3), 379-384. нтTрs:// DOI.ORG/10.1016/J.AMEPRE.2016.10.008

Lozano, F. y Domingo, P. (2011). Tratamiento antirretroviral de la infección por el VIH. Enfermedades Infecciosas y Microbiología Clínica, 29(6), 455-465. HTTPS://DOI.ORG/10.1016/ J.EIMC.2011.02.009

Martínez, M., Hernández, M. y Hernández, M. (2006). Psicometría. Alianza Editorial.

Mendoza, A., Flores, H. y Martínez, L. (2013). Factores que intervienen en el uso correcto y sistemático del condón, relacionados con la percepción de riesgo, según estudiantes universitarios panameños. Revista Centroamericana de Obstetricia $y$ Ginecología, 18(1), 21-25. HTTPS://DOI.ORG/ 10.37980/IM.JOURNAL.REVCOG.1637 
Miguel-Tobal, J. (2003). Cuestionarios, inventarios y escalas. En F. Labrador, J. Cruzado y M. Muñoz (Eds.), Manual de técnicas de modificación y terapia de conducta (pp. 151-179). Pirámide.

Milic, M., Gazibara, T., Stevanovic, J., Parlic, M., Nicholson, D., Mitic, K., Lazic, D. y Dotlic, J. (2020). Patterns of condom use in a university student population residing in a high-risk area for HIV infection. The European Journal of Contraception $y$ Reproductive Health Care, 25(4), 269-278. HTTPs:// DOI.ORG/10.1080/13625187.2020.1766674

Montero, I. y León, O.G. (2007). A guide for naming research studies in psychology. International Journal of Clinical and Health Psychology, 7, 847- 862

Muñiz, J. (1998). La medición de lo psicológico. Psicothema, 10(1), 1-21.

Nunnally, J. y Bernstein, I. (1995). Teoría psicométrica. Mc Graw-Hill.

Olansky, E., Mansergh, G., Pitts, N., Mimiaga, M., Denson, D., Landers, S., Holman, J. y Herbst, J. (2020). PrEP awareness in the context of HIV/ AIDS conspiracy beliefs among black/african american and hispanic/latino MSM in three urban us cities. Journal of Homosexuality, 67(6), 833-843. H T T P S : / / D O I . O R G / 10.1080 00918369.2018 .1557953

Olaz, F. (2014). Confiabilidad. En S. Tornimbeni, E. Pérez y F. Olaz (Eds), Introducción a la psicometría (pp. 71-100). Paidós.

OMS [Organización Mundial de la Salud] (2019). VIH/Sida. HTTPS://Www.WHO.INT/ES/NEWSROOM/FACT-SHEETS/DETAIL/HIV-AIDS

OPS [Organización Paramericana de la Salud] (2020). 136 mil personas viven con VIH en Argentina. HTTPS://WWW.PAHO.ORG/ES/NOTICIAS/1-12-2020136-MIL-PERSONAS-VIVEN-CON-VIH-ARGENTINA

Otzen, T. y Manterola, C. (2017). Técnicas de muestreo sobre una población de estudio. International Journal of Morphology, 35(1), 227-232. HTTPs:// DOI.ORG/10.4067/s0717-95022017000100037

ONUSIDA [Programa Conjunto de las Naciones Unidas sobre el VIH/sida] (2019). Monitoreo Global del SIDA 2020. Indicadores para el seguimiento de la Declaración Política de las Naciones Unidas para poner fin al SIDA de 2016. HTTPS://WWW.UNAIDS.ORG/SITES/DEFAULT/FILES/ MEDIA_ASSET/GLOBAL-AIDS-MONITORING_ES.PDF

ONUSIDA [Programa Conjunto de las Naciones Unidas sobre el VIH/sida] (2021). Hoja informativa 2021. Estadísticas epidemilógicas preeliminares mundiales de ONUSIDA para 2021. HTTPS://WWW.UNAIDS.ORG/SITES/DEFAULT/FILES/ MEDIA_ASSET/UNAIDS_FACTSHEET_ES.PDF
Pavía-Ruz, N., Góngora-Biachi, R., Vera-Gamboa, L., Moguel-Rodríguez, W. y González-Martínez, P. (2012). Conocimientos, actitudes y percepción de riesgo en referencia al VIH/SIDA en población rural de Yucatán, México. Revista Biomédica, 23(2), 53-60. HTTPS://DOI.ORG/10.32776/ REVBIOMED.V23I2.86

Pérez, E. y Tornimbeni, S. (2014). Construcción de tests. En S. Tornimbeni, E. Pérez y F. Olaz (Eds), Introducción a la psicometría (pp. 161-190). Paidós.

Posada, I., Yepes, C. y Patiño, L. (2020). Amor, riesgo y SIDA: hombres que tienen sexo con hombres. Estudos Feministas, Florianópolis, 28(1), 1-13. H T TPS:// D OI.ORG/10.1590/1806-95842020V28N150443

Reidl-Martínez, L. (2013). Confiabilidad en la medición. Investigación en Educación Médica, 2(6), 107-111. HTTPS://DOI.ORG/10.1016/s2007$5057(13) 72695-4$

Rosales, C. (2019). ¿De qué temas sexuales hablan y cómo negocian el uso del condón hombres que tienen sexo con hombres? REDES. Revista de Divulgación Crisis y Retos en la Familia y Pareja, 1(1), 6-10. HTTPS://DOI.ORG/10.22402/ J.REDES.UNAM.1.1.2019.229.6-10

Saeteros, R., Pérez, J. y Sanabria, G. (2015). Conducta de riesgo y problemas sexuales y reproductivos de estudiantes universitarios ecuatorianos. Humanidades Médicas, 15(3), 421-439.

Salvador-Ginez, O. y Ortega-Andeane, P. (2018). Mexico's approach to disasters in the 21st century: A Trans-disciplinary and multimethod perspective from environmental psychology. En J.C. PenagosCorzo y M.A. Padilla (Eds.), Challenges in creativity and psychology for the XXI century (pp. 312-328). UDLAP-UDG.

Sánchez, R., Lozano, M., Negrete, O., Enríquez, D., y Estrada, M. (2021). Base de datos sobre percepción de riesgo ante el VIH en hombres [Data set]. Zenodo. HTTP://DOI.ORG/10.5281/ZENODO.5008989

Slovic, P. (1987). Perception of risk. Science, 236(4799), 280-285. HTTPS://DOI.ORG/10.1126/ SCIENCE. 3563507

Stanojlovic, M. (2015). Percepción social de riesgo: una mirada general y aplicación a la comunicación de salud. Revista de Comunicación y Salud, 5, 99-110. H T T P S: / / D O I. O R G / 10.35669 / REVISTADECOMUNICACIONYSALUD.2015.5(1).99-110

Uribe, A, Castellanos, J., y Cabán, M. (2016). Conductas sexuales de riesgo y comunicación sobre sexualidad entre padres e hijos universitarios. Revista de Psicología Universidad de Antioquia, 8(2), 27-48. HTTPs://DOI.ORG/ 10.17533/UDEA.RPSUA.V8N2AO3 
Vallejo, P. (2011). El análisis factorial en la construcción $e$ interpretación de tests, escalas y cuestionarios. Universidad Pontificia Comillas.

Vinaccia, S., Quinceno, J., Gaviria, A., Soto, A., Gil, M. y Ballester, R. (2007). Conductas sexuales de riesgo para la infección por VIH/SIDA en adolescentes colombianos. Terapia Psicológica, 25(1), 39-50. H T TPS:// D OI.ORG/ $10.4067 /$ SO 718 48082007000100003

Yates, F. y Stone, E. (1992). The risk construct. En F. Yates (Ed.), Risk taking behavior (pp.1-25). Wiley. 